\title{
Flora e aspectos auto-ecológicos de um encrave de cerrado na chapada do Araripe, Nordeste do Brasil ${ }^{1}$
}

\author{
Itayguara Ribeiro da Costa ${ }^{2}$, Francisca Soares de Araújo ${ }^{3,4}$ e Luiz Wilson Lima-Verde ${ }^{3}$
}

Recebido em 15/06/2002. Aceito em 08/04/2004

\begin{abstract}
RESUMO - (Flora e aspectos auto-ecológicos de um encrave de cerrado na chapada do Araripe, Nordeste do Brasil). Este trabalho visa conhecer a composição e riqueza florística, os padrões fenológicos reprodutivos, as síndromes de dispersão e as formas de vida das espécies de uma disjunção de cerrado em clima semi-árido, na chapada do Araripe, Estado do Ceará. Foram encontradas 107 espécies e 41 famílias. Fabaceae, Myrtaceae, Poaceae, Apocynaceae, Euphorbiaceae e Malpighiaceae foram as mais ricas em espécies. Foi feita a distribuição geográfica de 47 espécies arbustivas e arbóreas em 27 listagens de cerrados brasileiros. Doze espécies apresentaram ampla distribuição geográfica e 13 foram registradas apenas neste trabalho. Cerca de 76\% das espécies floresceram e frutificaram no período chuvoso. As síndromes de dispersão predominantes foram: zoocoria, autocoria e anemocoria. O espectro biológico foi predominantemente constituído por fanerófitos (50,7\%), hemicriptófitos (14,9\%) e caméfitos (13,1\%). O cerrado estudado apresentou menor riqueza taxonômica que os cerrados contínuos e comportamento das fenofases reprodutivas, percentagem de síndromes de dispersão e formas de vida similares.
\end{abstract}

Palavras-chave: cerrado, flora vascular, dispersão, fenologia, formas de vida

\begin{abstract}
Flora and autecology's aspects of a disjunction cerrado at Araripe plateau, Northeastern Brazil). This study subject to investigate the floristic composition and richness, the reproductive phenological patterns, the dispersal syndromes and life forms of species of a disjunt cerrado in semiarid climate at Araripe plateau during a one year period. We found 107 species and 41 families. Fabaceae, Myrtaceae, Poaceae, Apocynaceae, Euphorbiaceae and Malpighiaceae showed the largest number of species. For 47 of the woody species found, we studied the geographical distribution based on 27 papers of the Brazilian cerrados. Twelve species are of widespread occurence in the cerrado, and 13 are restricted to the Araripe plateau. Zoocory, autocory, and anemocory are the predominant syndromes of dispersal. The predominant life forms were phanerophytes (50.7\%), hemicriptophytes (14.9\%) and camephytes (13.1\%). The cerrado of Araripe have lower species richness than continous cerrados, but a similar pattern of reproductive phenology, dispersal syndromes and life forms in more humid zones.
\end{abstract}

Key words: cerrado, vascular flora, dispersal syndromes, phenology, life forms

\section{Introdução}

O cerrado é a segunda maior formação vegetacional do Brasil, cobrindo cerca de $23 \%$ do território ou aproximadamente 2 milhões de $\mathrm{km}^{2}$ (Furley \& Ratter 1988; Ratter et al. 1997). Sua área core cobre o planalto Central, estendendo-se até o Piauí e Maranhão, ocorrendo também em forma de disjunções nas regiões Norte e Nordeste e nos Estados de São Paulo e Paraná (Eiten 1972). A área core do cerrado em geral está submetida a estacionalidade climática, cujo período seco, de maio a agosto, coincide com os meses mais frios do ano (Furley \& Ratter 1988). Entretanto, devido à sua extensão territorial, esta vegetação é encontrada sob grande variação climática, desde precipitação média anual de menos de $800 \mathrm{~mm}$, no Nordeste do Brasil, a mais de 2.000mm no extremo Noroeste do Brasil, e temperatura média anual de $20^{\circ}$ a $25^{\circ} \mathrm{C}$ (Furley \& Ratter 1988). Essas variações climáticas (Furley \& Ratter 1988), associadas a fatores edáficos e à ação do fogo (Eiten 1972; Coutinho 1978), topografia e drenagem (Furley \& Ratter 1988) são refletidas na grande variação fisionômica do cerrado. Conseqüentemente, a riqueza e a diversidade florística também variam. Castro et al. (1999) estimaram que o cerrado teria de 3.000 a 7.000 espécies lenhosas, apresentando padrão de variação lati-altitudinal. Porém, quando considerado apenas o estrato arbustivo-

\footnotetext{
1 Auxílio parcial da Fundação O Boticário de Proteção à Natureza

2 Programa de Pós-Graduação em Biologia Vegetal / UNICAMP, Cidade Universitária Zeferino Vaz, s/n., C. Postal 6109, CEP 13083-970, Campinas, SP, Brasil (itayguara@yahoo.com)

3 Departamento de Biologia, Universidade Federal do Ceará, Campus do Pici, Bloco 906, CEP 60455-760, Fortaleza, CE, Brasil

4 Autor para correspondência: tchesca@ufc.br
} 
arbóreo, segundo Ratter et al. (1997) a diversidade alfa, em geral, não ultrapassa a 120 espécies por hectare, sendo menor ainda nas áreas disjuntas, como as localizadas na Amazônia.

Como adaptação à sobrevivência durante o período de maior estiagem, algumas espécies apresentam formas de vida temporárias como hemicriptófitos, terófitos e geófitos (Mantovani \& Martins 1988; Batalha et al. 1997). Além disso, também ocorrem espécies que apresentam padrões fenológicos reprodutivos e síndromes de dispersão ajustados à estacionalidade climática. As espécies do estrato herbáceo florescem e frutificam ao final da estação chuvosa, enquanto as arbustivas e arbóreas o fazem no início da mesma (Mantovani \& Martins 1988; Batalha et al. 1997; Batalha \& Mantovani 2000). As espécies anemocóricas, principalmente do estrato herbáceo, dispersam seus frutos na estação seca, enquanto as espécies zoocóricas, principalmente do estrato arbustivo-arbóreo, o fazem na estação chuvosa (Batalha et al. 1997; Batalha \& Mantovani 2000; Weiser \& Godoy 2001).

No Nordeste do Brasil, as disjunções de cerrado ocorrem principalmente em baixas altitudes, de 0 a 500m (Castro 1999), sobre os tabuleiros costeiros (Formação Barreiras), nos Estados de Pernambuco (Sarmento \& Soares 1971), Paraíba (Tavares 1988; Oliveira-Filho \& Carvalho 1993) e Ceará (Figueiredo 1989; 1997; Fernandes 1990). Porém, além dos que ocorrem na Formação Barreiras, no Ceará são encontrados outros encraves: a) no sul do Estado, nos municípios de Iguatu, Salgado e nas serras de Caririaçu e chapada do Araripe e b) no norte, na porção norte do planalto da Ibiapaba (Figueiredo 1989; 1997; Fernandes 1990). O cerrado da chapada do Araripe e do planalto da Ibiapaba ocorrem em altitudes superiores a 800m (Figueiredo 1997).

No Estado do Ceará, a única área de cerrado preservada está situada na chapada do Araripe, dentro da área da Floresta Nacional do Araripe (FLONA Araripe), correspondendo a 10.618,75ha, cerca de $27,5 \%$ da área total da FLONA-Araripe (www.ibama.gov.br/recursosflorestais/araripe).

O cerrado da chapada do Araripe é uma disjunção situada a altitude de 800 a 900m, encravado no domínio semi-árido da caatinga. Devido à maior altitude e, conseqüentemente, sob maior precipitação e menor temperatura que na caatinga do entorno, esse encrave caracteriza-se como um hábitat ilha. Portanto, é esperado que o cerrado da chapada do Araripe, ilhado e inserido em região de clima mais árido que o da área core, apresente menor riqueza de táxons que os cerrados do planalto central e seja constituído por espécies de ampla distribuição geográfica.

Para responder esta hipótese e considerando o que aponta o MMA (1999), neste trabalho propôs-se o conhecimento da composição, a riqueza e a distribuição da flora e a identificação de algumas características ecológicas das espécies, tais como: padrões fenológicos reprodutivos, síndromes de dispersão e formas de vida, numa área de cerrado na chapada do Araripe, Ceará.

\section{Material e métodos}

Área de estudo - O levantamento florístico foi realizado em área de 10ha de cerrado sensu stricto situada a nordeste da chapada Araripe $\left(07^{\circ} 24^{\prime}\right.$ 'S e $39^{\circ} 20^{\prime} \mathrm{W}$ e 900m de altitude), dentro da área da Floresta Nacional do Araripe (FLONA-Araripe), no município de Barbalha, Ceará. O solo é constituído por associação de Latossolos Vermelho-Distróficos (Jacomine et al. 1973). No posto pluviométrico da Fundação Cearense do Estado do Ceará (FUNCEME), localizado na sede do Município de Jardim, ao sul da chapada ( $07^{\circ} 35^{\prime}$ 'S e $\left.39^{\circ} 17^{\prime} \mathrm{W}, 630 \mathrm{~m}\right)$, a precipitação média é de $759 \mathrm{~mm}$. ano $^{-1}$, com $66,3 \%$ do total concentrados entre os meses de janeiro a abril (Fig. 1). A temperatura média anual estimada por regressão foi de $24,1^{\circ} \mathrm{C}$ (SUDENE 1982), oscilando de $22,1^{\circ} \mathrm{C}$ no mês mais frio (julho) a $25,8^{\circ} \mathrm{C}$ no mês mais quente (novembro) (Fig. 1). A precipitação total do período de estudo, no posto de Jardim foi de 206,8mm (FUNCEME 2002), abaixo da média, que é de $758 \mathrm{~mm}$.

Levantamento florístico - Foram realizadas coletas mensais, durante o período de julho/2000 a junho/2001

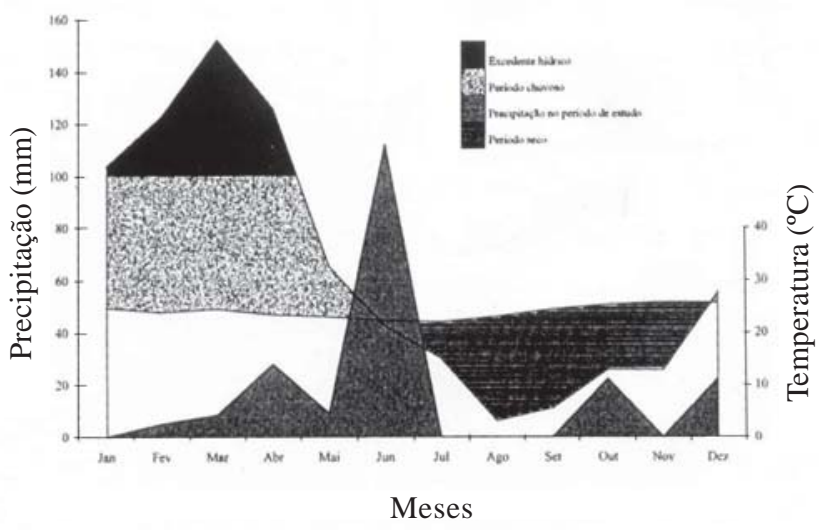

Figura 1. Gráfico ombrotérmico com as médias mensais de precipitação do período de 1978 a 2002 e precipitação mensal no período de estudo no posto de Jardim, Município de Jardim, Ceará. A temperatura média foi estimada por regressão. 
e o material coletado foi herborizado, identificado com o auxílio de chaves dicotômicas para gêneros e espécies e/ou por comparação no Herbário EAC (Prisco Bezerra) da Universidade Federal do Ceará, ou por envio direto a especialistas. Todo o material coletado foi depositado no Herbário EAC. A nomenclatura utilizada para denominação das famílias seguiu o sistema de Cronquist (1981), exceto para o grupo das Fabaceae (Leguminosae), que aqui foi tratado como uma única família.

Distribuição das espécies arbustivas e arbóreas - Foi feita a distribuição dos táxons identificados até o nível de espécie, com base em 27 listagens de cerrado: oito levantamentos na região Norte (Egler 1960; Takeuchi 1960; Rodrigues 1971; Dantas \& Rodrigues 1982; Bastos 1984; Gottsberger \& Moravetz 1986; Miranda 1993; Sanaiotti et al. 1997), oito no Sudeste (O. Cavassan, dados não publicados; A.A.J.F. Castro, dados não publicados; Cesar et al. 1988; Felfili \& Silva Jr. 1993; Araújo et al. 1997; Batalha \& Mantovani 2000; Costa \& Araújo 2001), sete no Centro-Oeste (Filgueiras 1990; Felfili \& Silva Jr. 1993; Nascimento \& Saddi 1992; Maury et al. 1994), três no Nordeste (Figueiredo 1989; Oliveira-Filho \& Carvalho 1993; Castro et al. 1998) e um no Sul (Uhlmann 1998). Foram consideradas espécies de ampla distribuição as que, além de ocorrerem no planalto central, também foram observadas em pelo menos outras duas das regiões acima citadas.

Padrões fenológicos reprodutivos e síndromes de dispersão - As fenofases floração e frutificação foram determinadas com base no material botânico coletado. Quando este não foi coletado, fez-se anotações na caderneta de campo sobre presença da fenofase na espécie. Na fenofase floração foram considerados todos os estádios, desde o botão floral até a antese da flor. $\mathrm{Na}$ fenofase frutificação foram considerados os estádios desde frutos imaturos até prontos para dispersão.

A caracterização das síndromes de dispersão foi baseada nas características morfológicas dos diásporos (frutos ou sementes) seguindo a nomenclatura proposta por Pijl (1982). Quando as espécies não foram encontradas com frutos, seguiu-se informações existentes para as espécies nos trabalhos de Mantovani \& Martins (1993), Batalha \& Mantovani (2000) e Weiser \& Godoy (2001).

Formas de vida - A classificação das espécies por forma de vida com hábitat terrestre foi feita segundo o sistema de Rankiauer (1934). Quando a espécie apresentou indivíduos ora caméfitos, ora nanofanerófitos, foi acrescentada a forma intermediária fanerófito-caméfito, como proposto por Weiser \& Godoy (2001). As epífitas e hemi-parasitas foram classificadas em separado, como nos trabalhos de Mantovani \& Martins (1993), Batalha \& Mantovani (2000) e Weiser \& Godoy (2001).

\section{Resultados}

Levantamento florístico - Nos 10 ha foram encontradas 107 espécies, distribuídas em 41 famílias e 85 gêneros (Tab. 1). Fabaceae foi a família com maior riqueza de espécies (17), seguida de Myrtaceae e Poaceae com oito, Euphorbiaceae e Malpighiaceae com cinco, Apocynaceae e Polygalaceae com quatro espécies. As famílias Annonaceae, Asteraceae, Solanaceae e Verbenaceae foram representadas por três espécies cada, enquanto 28 famílias (68\%) ocorreram com até duas espécies.

As famílias com maior riqueza de gêneros foram Fabaceae (14), seguida de Poaceae (sete), Euphorbiaceae (quatro), Malpighiaceae e Myrtaceae (três). Cerca de 51\% das famílias (21) foram representadas por um único gênero. Os gêneros com maior riqueza de espécies foram Psidium (quatro), Byrsonima e Solanun (três), sendo que 15 gêneros (17,6\% do total) foram representados por duas espécies e 67 gêneros (78,8\%) por uma única espécie.

O componente arbustivo-arbóreo foi representado por 60 espécies (56\%) e 28 famílias e o subarbustivoherbáceo por 48 espécies (44\%) e 23 famílias. No componente arbustivo e arbóreo destacaram-se as famílias Fabaceae (10 espécies), Myrtaceae (sete), Polygalaceae (quatro) e Malpighiaceae (três), e no componente herbáceo as de maior riqueza foram Poaceae (oito), Fabaceae (sete), Cyperaceae (quatro) e Asteraceae (três).

Distribuição da flora do componente arbustivoarbóreo - De 47 táxons identificados até o nível de espécie, 12 (20\%) apresentaram ampla distribuição geográfica, oito foram registradas somente na área core, quatro só nos cerrados de baixa altitude, amazônicos e nordestinos, cinco foram exclusivas dos cerrados nordestinos, ocorrendo nos Estados do Ceará, Paraíba e Piauí, e 13 (28\%) das espécies não foram encontradas em nenhuma das 27 listagens de cerrado analisadas (Tab. 2).

Padrões fenológicos reprodutivos e síndromes de dispersão - Na estação seca, durante os meses de julho 
Tabela 1. Lista das famílias e espécies coletadas no cerrado da chapada do Araripe, Barbalha, CE, com os respectivos hábitos, formas de vida, síndromes de dispersão, período de floração e frutificação, e números de coletor. Hábito: herb - herbáceo, arb - arbustivo, árv arbóreo, sub - subarbustivo, hemip - hemiparasita, trep - trepadeira, lia - liana e epif - epífita. Formas de vida: nano - nanofanerófitos, micro - microfanerófitos, meso - mesofanerófitos, cam - caméfito, fan-cam - fanerófito-caméfito, hemi - hemicriptófito, geo - geófito, tero - terófito. Síndromes de dispersão: zoo - zoocoria, ane - anemocoria, aut - autocoria. Período de floração e frutificação: jan - janeiro, fev - fevereiro, mar - março, abr - abril, mai - maio, jun - junho, jul - julho, ago - agosto, set - setembro, out - outubro, nov - novembro, dez - dezembro. (-) ausência da fenofase no período de estudo.

\begin{tabular}{|c|c|c|c|c|c|c|c|}
\hline Família/Espécies & Nome popular $\mathrm{I}$ & Hábito & $\begin{array}{l}\text { Forma de } \\
\text { vida }\end{array}$ & $\begin{array}{l}\text { Síndrome de } \\
\text { dispersão }\end{array}$ & $\begin{array}{l}\text { Período de } \\
\text { floração }\end{array}$ & $\begin{array}{ll}\text { Período de } \mathrm{N} . \\
\text { frutificação } \mathrm{Co}\end{array}$ & $\begin{array}{l}\text { N. coletor: } \\
\text { Costa, IR }\end{array}$ \\
\hline \multicolumn{8}{|l|}{ AMARANTHACEAE } \\
\hline Alternanthera brasiliana (L.) Kuntze & quebra-panela & herb & tero & auto & jun & jun & 33 \\
\hline Gomphrena sp. & & herb & hemi & auto & jun & jun & 31 \\
\hline \multicolumn{8}{|l|}{ ANACARDIACEAE } \\
\hline Anacardium microcarpum Ducke & cajuí & árv & micro & zoo & out & out & 72 \\
\hline \multicolumn{8}{|l|}{ ANNONACEAE } \\
\hline Annona coriacea Mart. & pinha & arb & fan-cam & $\mathrm{zoO}$ & out-jan & fev & 86 \\
\hline A. tomentosa R.E. Fr. & pinha & arb & nano & $\mathrm{zOO}$ & jan & - & 172 \\
\hline $\begin{array}{l}\text { Duguetia furfuracea (A. St-Hil.) } \\
\text { Benth. \& Hook. }\end{array}$ & pinha-brava & arb & fan-cam & $\mathrm{zoO}$ & jun, jan & jan & 23 \\
\hline \multicolumn{8}{|l|}{ APOCYNACEAE } \\
\hline Allamanda cf. puberula A.DC. & & trep & cam & ane & jun & - & 304 \\
\hline Hancornia speciosa Gomez & mangaba & árv & micro & zoo & mar, jun & dez-jan, mar, jun & un \\
\hline Himatanthus drasticus (Mart.) Plumel & janaguba & arb & micro & ane & nov & jun & 24 \\
\hline $\begin{array}{l}\text { Mandevila cf. velutina (Mart. ex Stadelm.) } \\
\text { Woodson }\end{array}$ & & trep & cam & ane & dez-fev & fev & 187 \\
\hline \multicolumn{8}{|l|}{ ASCLEPIADACEAE } \\
\hline \multicolumn{8}{|l|}{ ASTERACEAE } \\
\hline Gochnatia blanchetiana (DC.) Cabrera & & herb & hemi & ane & abr & abr & 44 \\
\hline Vanilosmopsis arborea (Gardner) Backer & candieiro & árv & micro & ane & ago & ago & 331 \\
\hline Vernonia araripensis Gardner & & herb & hemi & ane & jun & jun & 17 \\
\hline \multicolumn{8}{|l|}{ BIGNONIACEAE } \\
\hline Pyrostegia venusta Miers & cipó-de-são-joão & trep & cam & ane & jun & - & 314 \\
\hline \multicolumn{8}{|l|}{ BROMELIACEAE } \\
\hline Aechmaea bromeliifolia (Rudge) Baker & & epif & epif & ane & dez & dez, jun & 117 \\
\hline \multicolumn{8}{|l|}{ CARYOCARACEAE } \\
\hline Caryocar coriaceum Wittm. & pequi & árv & micro & $\mathrm{zoO}$ & set-nov & jan-mar & 61 \\
\hline \multicolumn{8}{|l|}{ CHRYSOBALANACEAE } \\
\hline Hirtella gracilipes (Hook. f.) Prance & chorão & árv & micro & zoo & out & nov & 256 \\
\hline H. ciliata Mart. \& Zucc. & chorão & árv & micro & $\mathrm{zOO}$ & abr & - & 108 \\
\hline \multicolumn{8}{|l|}{ CUSCUTACEAE } \\
\hline \multicolumn{8}{|l|}{ CLUSIACEAE } \\
\hline Vismia guianensis Aubl. & lacre-vermelho & arb & micro & zoo & jun, ago, dez & dez-jan & 51 \\
\hline \multicolumn{8}{|l|}{ CYPERACEAE } \\
\hline Cyperus cf. ligularis L. & & herb & geo & auto & jan & jan & 148 \\
\hline Rhynchospora exaltata Kunth & & herb & geo & auto & mar & mar & 3 \\
\hline R. nervosa Boeck. & & herb & geo & auto & fev & fev & 194 \\
\hline Scleria secans (L.) Urb. & capim navalha & herb & geo & auto & dez & dez & 129 \\
\hline \multicolumn{8}{|l|}{ EBENACEAE } \\
\hline $\begin{array}{l}\text { Diospyros coccolobaefolia Mart. ex Miq. } \\
\text { ERYTHROXYLACEAE }\end{array}$ & olho-de-boi & árv & nano & zoo & nov & jan-mar & 88 \\
\hline Erythroxylum lofgrenii Diogo & carrasco & sub & cam & $\mathrm{zoO}$ & out, jan & jan & 139 \\
\hline E. barbatum O.E. Schulz & carrasco & arb & nano & zoo & mai & jun & 64 \\
\hline \multicolumn{8}{|l|}{ EUPHORBIACEAE } \\
\hline Croton sonderianus Müll. Arg. & marmeleiro-branco & arb & nano & zoo & fev-abr & fev-abr & 225 \\
\hline C. rhamifolius Kunth & marmeleiro & arb & nano & zoo & abr & abr & 159 \\
\hline Manihot palmata Müll. Arg. & maniçoba & arb & micro & auto & jun & jun & $\begin{array}{c}345 \\
\text { continua }\end{array}$ \\
\hline
\end{tabular}


Tabela 1 (continuação)

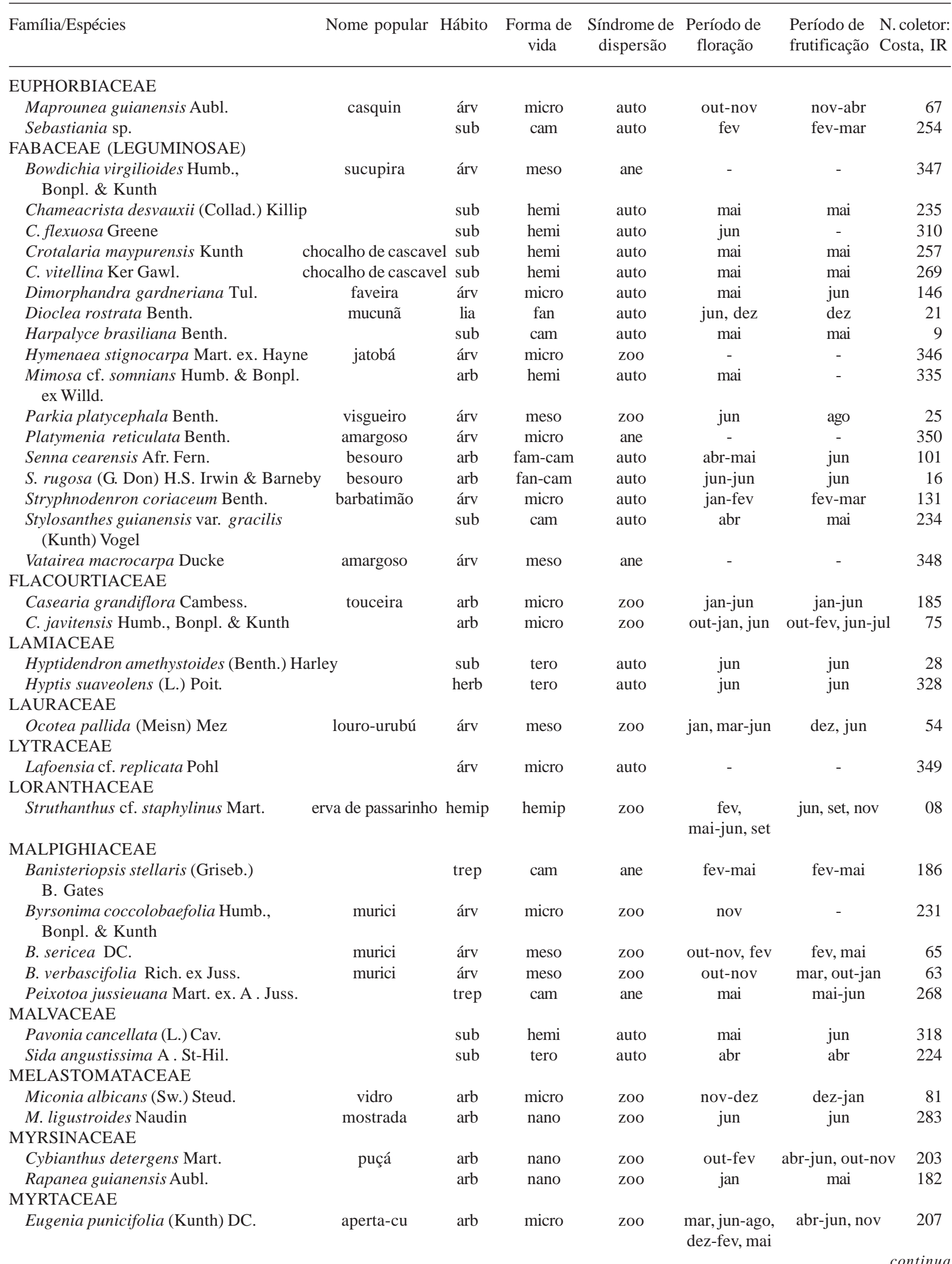


Tabela 1 (continuação)

\begin{tabular}{|c|c|c|c|c|c|c|c|}
\hline Família/Espécies & Nome popular & Hábito & $\begin{array}{l}\text { Forma de } \\
\text { vida }\end{array}$ & $\begin{array}{c}\text { Síndrome de } \\
\text { dispersão }\end{array}$ & $\begin{array}{l}\text { Período de } \\
\text { floração }\end{array}$ & $\begin{array}{l}\text { Período de } \\
\text { frutificação }\end{array}$ & $\begin{array}{l}\text { N. coletor } \\
\text { Costa, IR }\end{array}$ \\
\hline \multicolumn{8}{|l|}{ MYRTACEAE } \\
\hline Eugenia sp. & & sub & hemi & zoo & jan & mar & 341 \\
\hline Myrcia multiflora ( Lam.) DC. & cambuí & arb & nano & zoo & nov & dez-mar & 76 \\
\hline M. rostrata DC. & & arb & nano & zoo & jan, mar, jun, set & - & 1 \\
\hline Psidium cf. aerugineum O. Berg & & árv & micro & $\mathrm{zoO}$ & - & - & 348 \\
\hline P. myrsinoides O. Berg & & árv & micro & zoo & - & - & 275 \\
\hline P. pohlianum O. Berg & goiabinha & árv & micro & zoo & dez & mai & 238 \\
\hline P. suffruticosum O. Berg & & árv & micro & zoo & - & - & 272 \\
\hline \multicolumn{8}{|l|}{ OCHNACEAE } \\
\hline Ouratea parviflora Baill. & & arb & nano & zoo & ago-out & nov-jan & 49 \\
\hline \multicolumn{8}{|l|}{ ORCHIDACEAE } \\
\hline Catasetum barbatum Lindl. & & epif & cam & ane & ago & ago & 279 \\
\hline \multicolumn{8}{|l|}{ POACEAE } \\
\hline Aristida longifolia Trin. & & herb & hemi & zoo & jun & jun & 294 \\
\hline Asistida sp. & & herb & hemi & zoo & jun & jun & 295 \\
\hline Axonopus sp. & & herb & hemi & auto & abr & abr & 216 \\
\hline Eragostris maypurensi (Kunth) Steud. & & herb & hemi & zoo & jun & jun & 38 \\
\hline Gymnospogon poliasy (Willd.) F. Voes & & herb & tero & ane & jun & jun & 32 \\
\hline Panicum sp. & & herb & tero & auto & abr & abr & 229 \\
\hline Setaria sp. & & herb & hemi & zoo & ago & ago & 50 \\
\hline Trachypogon spicatus (L.f.) Kuntze & capim agulha & herb & hemi & ane & abr & abr & 215 \\
\hline \multicolumn{8}{|l|}{ POLYGALACEAE } \\
\hline $\begin{array}{l}\text { Bredemeyera brevifolia Klotzsch } \\
\text { ex A.W. Benn. }\end{array}$ & & árv & micro & ane & - & nov & 329 \\
\hline B. floribunda Willd. & & árv & micro & ane & jan & fev-mar & 151 \\
\hline Polygala aefridii Chodat & & arb & tero & ane & mai & jun & 232 \\
\hline P. spectabilis DC. & & arb & tero & ane & jan & - & 333 \\
\hline \multicolumn{8}{|l|}{ RUBIACEAE } \\
\hline $\begin{array}{l}\text { Alibertia myrciifolia (Spruce ex K. } \\
\text { Schum.) K. Schum. }\end{array}$ & batinga & arb & nano & zoo & set-nov & nov-jan & 58 \\
\hline $\begin{array}{l}\text { Tocoyena formosa (Cham. \& Schltdl.) } \\
\text { K. Schum. }\end{array}$ & jenipapo-bravo & árv & micro & zoo & jan & mai & 199 \\
\hline \multicolumn{8}{|l|}{ RUTACEAE } \\
\hline Zanthoxylum gardneri Engl. & laranjinha & arb & nano & zoo & dez-jan & jun-set & 128 \\
\hline \multicolumn{8}{|l|}{ SAPINDACEAE } \\
\hline Matayba guianensis Aubl. & pitomba & árv & micro & zoo & set & set-jan & 167 \\
\hline Serjania lethalis A. St-Hil. & & trep & cam & ane & set, jan, out & & 80 \\
\hline \multicolumn{8}{|l|}{ SAPOTACEAE } \\
\hline Chrysophyllum arenarium Allemão & ameixa & árv & micro & zoo & abr & ago-set & 218 \\
\hline \multicolumn{8}{|l|}{ SIMAROUBACEAE } \\
\hline Simarouba amara Aubl. & & árv & meso & zoo & nov & - & 68 \\
\hline \multicolumn{8}{|l|}{ SMILACACEAE } \\
\hline Smilax japicanga Griseb. & japecanga & trep & cam & zoo & - & nov, jan & 98 \\
\hline \multicolumn{8}{|l|}{ SOLANACEAE } \\
\hline Solanum cf. baturitense Huber & jurubeba & arb & cam & zoo & jan, abr, jun & jun & 292 \\
\hline $\begin{array}{l}\text { S. stipulaceum Willd. ex Roem. } \\
\text { \& Schult. }\end{array}$ & urubeba-de-espinho & o arb & cam & zoo & jun & jun & 293 \\
\hline Solanum sp. & jurubeba & sub & cam & $\mathrm{zoO}$ & jan & - & 346 \\
\hline \multicolumn{8}{|l|}{ VERBENACEAE } \\
\hline Amasonia coccinea Liebm. ex Moldenke & & herb & tero & auto & fev, abr & abr & 233 \\
\hline Lantana camara L. & chumbinho & herb & tero & auto & jun & jun & 11 \\
\hline Vitex schaueriana Moldenke & amargoso & árv & micro & zoo & - & - & 337 \\
\hline \multicolumn{8}{|l|}{ VISCACEAE } \\
\hline \multirow{2}{*}{\multicolumn{8}{|c|}{ VOCHYSIACEAE }} \\
\hline & & & & & & & \\
\hline Qualea parviflora Mart. & $\begin{array}{l}\text { pau-terra-da- } \\
\text { folha-miúda }\end{array}$ & árv & fan & meso & dez-jan & $\begin{array}{l}\text { nov-dez, } \\
\text { fev, mai-jun }\end{array}$ & 115 \\
\hline
\end{tabular}


Tabela 2. Distribuição das espécies do componente arbustivo-arbóreo do cerrado da chapada do Araripe Barbalha, CE, com base em outros trabalhos analisados por unidade da federação: AC: Takeuchi (1960), Egler (1960); AM - Gottsberger \& Moravetz (1986); AP - Sanaiotti et al (1997); CE - Figueiredo (1989); DF - Filgueiras \& Pereira (1990); Felfili \& Silva Jr. (1993), Felfili et al. (1993), Maury et al. (1994), GO - Felfili \& Silva Jr. (1993), Felfili et al. (1993); MG - Felfili \& Silva Jr. (1993), Felfili et al. (1993), Araújo et al. (1997), Costa \& Araújo (2001); MT - Nascimento \& Saddi (1992); PA - Bastos (1984), Miranda (1993); PB - Oliveira-Filho \& Carvalho (1993); PI - Castro et al. (1998); PR - Uhlmann et al. (1998); RR - Rodrigues (1971), Dantas \& Rodrigues (1982); SP - Cavassan (dados não publicados), Castro (dados não publicados), Cesar et al. (1988); Batalha \& Mantovani (2000).

Espécies

Alibertia mirciifolia (Spruce ex. K. Schum.) K. Schum. Anacardium microcarpum Ducke

Annona coriacea Mart.

Bowdichia virgilioides Humb., Bonpl. \& Kunth.

Bredemeyera brevifolia Klotzch ex A.W. Benn.

Bredemeyra floribunda Willd.

Byrsonima coccolobaefolia Humb., Bonpl. \& Kunth.

Byrsonima sericea DC.

Byrsonima verbascifolia Rich. ex Juss.

Caryocar coriaceum Wittm.

Casearia grandiflora Cambess.

Casearia javitensis Humb., Bonpl. \& Kunth.

Chrysophyllum arenarium Allemão

Cybianthus detergens Mart.

Dimorphandra gardneriana Tul.

Duguettia furfuracea (A. St-Hil.) Benth. \& Hook.

Eugenia punicifolia (Kunth) DC.

Hancornia speciosa Gomez

Himatanthus drasticus (Mart.) Plumel

Hymenaea stignocarpa Mart. ex Hayne

Hyrtella gracilipes (Hook. f.) Prance

Hyrtellla ciliata Mart. \& Zucc.

Maprounea guianensis Aubl.

Matayba guianensis Aubl.

Miconia albicans (Sw.) Steud.

Miconia ligustroides Naudin

Myrcia multiflora (Lam.) DC.

Myrcia rostrata DC.

Ocotea pallida (Meisn.) Mez

Ouratea parviflora (A. St-Hil.) Engl.

Parkia platycephala Benth.

Psidium myrsinoides O. Berg.

Psidium pohlianum O. Berg.

Psidium suffruticosum O. Berg.

Qualea parviflora Mart.

Rapanea guianensis Aubl.

Senna cearensis Afr. Fern.

Senna rugosa (G. Don) H.S. Irwin \& Barneby

Simarouba amara Aubl.

Solanum stipulaceum Willd ex Roen \& Schult.

Stryphnodenron coriaceum Benth.

Tocoyena formosa (Cham. \& Schltdl.) K. Schum.

Vanilosmopsis arborea (Gardner) Backer

Vatairea macrocarpa (Benth.) Ducke

Vismia guianensis Aulbl.

Vitex schaueriana Moldenke

Zanthoxylum gardneri Engler
Distribuição no Brasil por Unidades da Federação

PA

PA, CE

MG, SP, DF, GO, CE, PI,

MG, SP, MT, DF, GO, RR, AP, PA, PB, CE, PI

-

SP, DF, RR, PI,

MG, SP, DF, GO, MT, RR, PA, AP, PR,

PB, PI

MG, SP, MT, DF, GO, AP, AC, RR

CE, PI

MG, SP, DF, PA, AP

-

DF

CE, PI

SP, DF

MG, SP, PA, PB, PI

MG, SP, DF, GO, PA, AP, PB, CE, PI

-

MG, SP, MT, DF, GO, PI

MG, DF

AP, PB, PI

MG, SP, DF, AP

MG, DF, PA

MG, SP,DF, PI, AP

SP, DF

SP, DF

MG, SP, DF

-

CE, PI

MG, GO

-

MG, SP, MT, DF, GO, CE, PI

MG, SP, DF, PR, PB

-

SP, DF

DF, PA, AP

CE

MG, SP, MT, DF, PA, AP, AC, PI

-

MG, SP, DF, MT, PA, CE, PI

PA, AP, PB, PI

- 
Tabela 3. Trabalhos realizados em cerrados brasileiros com os respectivos critérios de inclusão, localização geográfica, precipitação e riqueza de espécies e famílias. PNS - perímetro no nível do solo, $\mathrm{P}_{10}$ - perímetro a $10 \mathrm{~cm}$ do solo, $\mathrm{P}_{30}$ - perímetro a $30 \mathrm{~cm}$ do solo, DBH - diâmetro a altura do peito, Dio - $10 \mathrm{~cm}$ do solo.

\begin{tabular}{|c|c|c|c|c|c|}
\hline \multirow[t]{2}{*}{ Autores/(Critérios de inclusão) } & \multirow[t]{2}{*}{ Local/Unidade da Federação } & \multirow{2}{*}{$\begin{array}{l}\text { Latitude } \\
\text { Longitude }\end{array}$} & \multirow{2}{*}{$\begin{array}{c}\text { Precipitação } \\
\text { (mm) }\end{array}$} & \multicolumn{2}{|c|}{ Número } \\
\hline & & & & Espécies & Famílias \\
\hline Este estudo (PNS $\geq 9 \mathrm{~cm}$ ) & FLONA Araripe/CE & $\begin{array}{l}07^{\circ} 24^{\prime} \mathrm{S} \\
29^{\circ} 20^{\prime} \mathrm{W}\end{array}$ & $\begin{array}{c}759 \\
758,7\end{array}$ & 59 & 30 \\
\hline Castro (1994) (PNS 9cm) & Chapada Grande/PI & $\begin{array}{l}06^{\circ} 36^{\prime} \mathrm{S} \\
42^{\circ} 16^{\prime} \mathrm{W}\end{array}$ & $1.226-1.230$ & 76 & 29 \\
\hline Oliveira Filho \& Carvalho (1993) Todos & Litoral Norte/PB & $\begin{array}{l}06^{\circ} 28^{\prime} \mathrm{S} \\
34^{\circ} 55^{\prime} \mathrm{W}\end{array}$ & 1.725 & - & - \\
\hline Castro (1987) (PNS $\geq 9 \mathrm{~cm})$ & P. E. do Vassununga/SP & $\begin{array}{l}21^{\circ} 38^{\prime} \mathrm{S} \\
47^{\circ} 36^{\prime} \mathrm{W}\end{array}$ & 1.524 & 83 & 35 \\
\hline Pagano et al. (1989) (Altura fuste $\geq 1,3 m$ ) & APA de Corumbataí/SP & $\begin{array}{l}22^{\circ} 08^{\prime} \mathrm{S} \\
47^{\circ} 40^{\prime} \mathrm{W}\end{array}$ & - & 123 & - \\
\hline Costa \& Araújo (2001) (PNS 15cm) & RPPN Panga/MG & $\begin{array}{l}19^{\circ} 09^{\prime} \mathrm{S} \\
48^{\circ} 23^{\prime} \mathrm{W}\end{array}$ & - & $76-93$ & $37-41$ \\
\hline Uhlmann et al. (1998) (PNS $\geq 15 \mathrm{~cm})$ & Parque Estadual do Cerrado/PR & $\begin{array}{l}24^{\circ} 09^{\prime} \mathrm{S} \\
50^{\circ} 18^{\prime} \mathrm{W}\end{array}$ & - & $18-33$ & $15-27$ \\
\hline Nascimento \& Saddi (1992) (D > 3cm) & Coxipó da Ponte/MT & $\begin{array}{l}15^{\circ} 36^{\prime} \mathrm{S} \\
56^{\circ} 06^{\prime} \mathrm{W}\end{array}$ & $750-2.000$ & $27-34$ & $14-17$ \\
\hline Felfili \& Silva Jr. (1993) $\left(\mathrm{P}_{30} \geq 5 \mathrm{~cm}\right)$ & EE Águas Emendadas/DF & $\begin{array}{l}\text { 153's } \\
47^{\circ} 32^{\prime} \mathrm{W}\end{array}$ & - & 72 & 31 \\
\hline & APA Gama Cabeça de Veado/DF & $\begin{array}{l}15^{\circ} 52^{\prime} \mathrm{S} \\
47^{\circ} 50^{\prime} \mathrm{W}\end{array}$ & - & 66 & 31 \\
\hline & PN Brasília/DF & $15^{\circ} 37^{\prime} \mathrm{S}$ & & & \\
\hline & Silvânia/GO & $\begin{array}{l}47^{\circ} 54^{\prime} \mathrm{W} \\
16^{\circ} 30^{\prime} \mathrm{S}\end{array}$ & - & 55 & 26 \\
\hline & Paracatu/MG & $\begin{array}{l}48^{\circ} 30^{\prime} \mathrm{W} \\
17^{\circ} 00^{\prime} \mathrm{S}\end{array}$ & - & 68 & 31 \\
\hline & & $46^{\circ} 45^{\prime} \mathrm{W}$ & - & 60 & 33 \\
\hline & Patrocínio/MG & $\begin{array}{l}18^{\circ} 47^{\prime} \mathrm{S} \\
46^{\circ} 25^{\prime} \mathrm{W}\end{array}$ & - & 68 & 32 \\
\hline Sanaiotti et al. (1997) (PAP $\geq 5 \mathrm{~cm})$ & 11 locais/AP & & $2.321-3.250$ & 61 & - \\
\hline Gottsberger \& Moravetz (1986) (Altura $\geq 2 \mathrm{~m}$ ) & Humaitá/AM & $\begin{array}{l}07^{\circ} 31^{\prime} \mathrm{S} \\
63^{\circ} 00^{\prime} \mathrm{W}\end{array}$ & 2.361 & 35 & 21 \\
\hline Miranda (1993) (Altura 1m) & Alter-do-Chão/PA & $\begin{array}{l}02^{\circ} 01^{\prime} \mathrm{S} \\
55^{\circ} 00^{\prime} \mathrm{W}\end{array}$ & 1.950 & 55 & 26 \\
\hline
\end{tabular}

a novembro, floresceram e/ou frutificaram cerca de 24\% das espécies coletadas, e durante o período chuvoso, dezembro a junho, o percentual de floração e/ou frutificação foi de 76\% (Fig. 2). Durante o período de estudo, foram verificados dois picos de floração. $\mathrm{O}$ primeiro pico (77\% das espécies) ocorreu no mês de outubro (Fig. 2), antes do início do período chuvoso (Fig. 1), e o segundo pico (52,7\%), ocorreu de janeiro a abril/2000 (Fig. 2), coincidindo com o período chuvoso (Fig. 1). Os picos de frutificação, em geral, ocorreram simultaneamente aos de floração, exceto no mês de outubro (Fig. 2).

Apenas oito espécies não produziram flores e nem frutos, ou pelo menos não foram encontradas férteis durante o período de estudo. Foram elas: Hymenaea stignocarpa, Vatairea macrocarpa, Lafoensia replicata, Platymenia reticulata, Psidium cf. aerigineum, $P$. myrsinoides, $P$. sufruticosum e Vitex cymosa. Entretanto sete espécies floresceram mais de uma vez durante o período de estudo: Byrsonima sericea, Casearia javitensis, Dioclea rostrata, Eugenia punicifolia, Hancornia speciosa, Serjania lethalis e Vismia guianensis (Tab. 1).

As síndromes de dispersão predominantes foram: zoocoria, em 53 espécies (49,5\%), seguida de autocoria, em $30(28,1 \%)$ e anemocoria em 24 espécies (22,4\%) (Tab. 1). Considerando apenas o componente arbustivo-arbóreo, zoocoria também foi a síndrome predominante $(71,6 \%)$, porém, no componente subarbustivo-herbáceo, dominaram as 


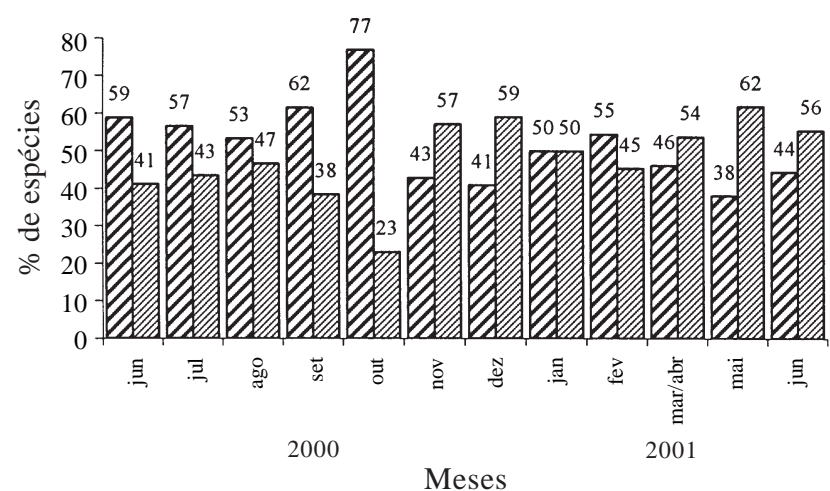

Figura 2. Variações mensais do número de espécies por fenofase, floração e frutificação, no período de julho/2000 a junho/2001. $\square=$ Flor; $\mathbb{Z}=$ Fruto.

plantas anemocóricas (53\%) e autocóricas (47\%) (Tab. 4).

A maioria das espécies com síndrome zoocórica produziu frutos durante todo o período de estudo, com o máximo de produção ocorrendo no mês de outubro (100\%), enquanto a maior proporção de espécies com síndrome anemocórica (60\%) ocorreu no início do período seco, em julho de 2000 e a maior percentagem de autocoria (38\%) foi no período anterior às chuvas, que neste estudo, foi entre os meses de março/abril (Fig. 3).

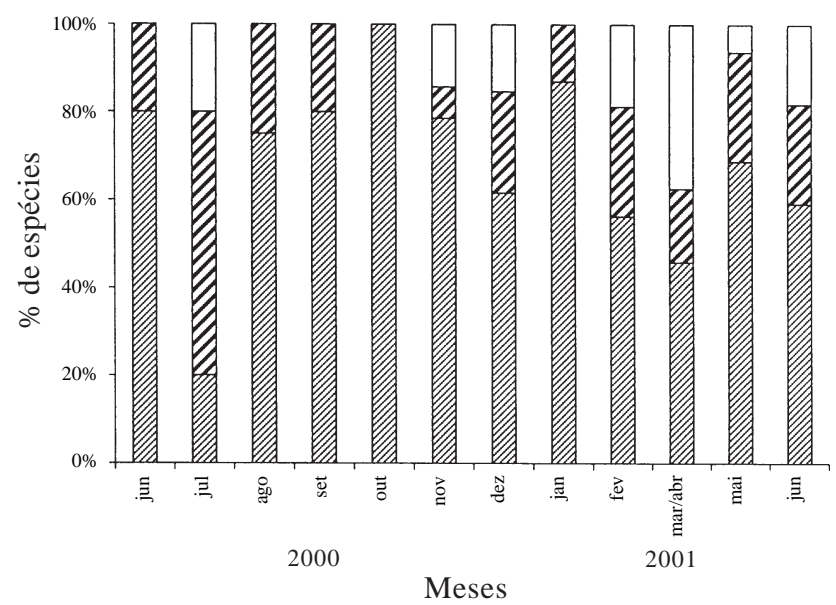

Figura 3. Variações mensais na percentagem de síndromes de dispersão das espécies encontradas com frutos no período julho/2000 a junho/2001. Ш/A =Zoocoórica; $\square$ =Anemocórica; $\square$ = Autocórica.

Tabela 4. Distribuição da percentagem de síndromes de dispersão encontradas neste estudo e em outros três levantamentos de cerrado em São Paulo. Total - percentagem total por levantamento; Comp. Arb - percentagem no componente arbustivo-arbóreo, Comp. Herb. - percentagem no componente herbáceo-subarbustivo.

\begin{tabular}{|c|c|c|c|c|c|c|c|c|c|}
\hline \multirow[b]{3}{*}{ Autores } & \multicolumn{9}{|c|}{ Síndromes de Dispersão (\%) } \\
\hline & \multicolumn{3}{|c|}{ Zoocoria } & \multicolumn{3}{|c|}{ Anemocoria } & \multicolumn{3}{|c|}{ Autocoria } \\
\hline & Total & $\begin{array}{l}\text { Comp. } \\
\text { Arb. }\end{array}$ & $\begin{array}{l}\text { Comp. } \\
\text { Herb. }\end{array}$ & Total & $\begin{array}{l}\text { Comp. } \\
\text { Arb. }\end{array}$ & $\begin{array}{l}\text { Comp. } \\
\text { Herb. }\end{array}$ & Total & $\begin{array}{l}\text { Comp. } \\
\text { Arb. }\end{array}$ & $\begin{array}{l}\text { Comp. } \\
\text { Herb. }\end{array}$ \\
\hline Este estudo & 49,5 & 79,2 & 20,8 & 22,4 & 25,0 & 75,0 & 28,1 & 26,6 & 73,4 \\
\hline Batalha et al. (1993) & 39,0 & 65,0 & 29,0 & 34,0 & 25,2 & 36,6 & 27,0 & 9,8 & 34,7 \\
\hline Batalha \& Mantovani (2000) & 37,5 & 62,0 & 25,9 & 34,3 & 25,9 & 38,1 & 28,2 & 12,0 & 36,9 \\
\hline Weiser \& Godoy (2001) & 43,9 & 64,0 & 23,0 & 33,1 & 27,0 & 38,0 & 23,0 & 9,0 & 39,0 \\
\hline
\end{tabular}

Tabela 5. Espectro biológico das espécies do cerrado da chapada do Araripe e de outros três levantamentos de cerrado em São Paulo. Fan - fanerófito, Cam - caméfito, Fan-Cam - fanerófito-caméfito, Hemi - hemicriptófito, Geo - geófito, Tero - terófito.

\begin{tabular}{|c|c|c|c|c|c|c|c|}
\hline \multirow[t]{2}{*}{ Levantamentos } & \multicolumn{7}{|c|}{ Formas de vida predominantes (\%) } \\
\hline & Fan & Cam & Fan-Cam & Hemi & Geo & Tero & Outros \\
\hline Este estudo & 50,7 & 13,1 & 3,7 & 14,9 & 3,7 & 9,3 & $\begin{array}{l}\text { Epífitos: } 2,8 \\
\text { Hemiparasitas: } 1,8\end{array}$ \\
\hline Mantovani \& Martins (1993) & 21,9 & 14,5 & 1,0 & 43,5 & 2,1 & 6,7 & $\begin{array}{l}\text { Lianas: } 7,6 \\
\text { Epífitos: } 0,8 \\
\text { Hemiparasitas: } 0,4 \\
\text { Hemicriptófitos-Caméfitos: } 0,2\end{array}$ \\
\hline Batalha et al. (1993) & 28,0 & 18,0 & & 35,0 & 1,0 & 6,0 & $\begin{array}{l}\text { Lianas: } 9,0 \\
\text { Epífitos: } 2,0 \\
\text { Hemiparasitas: } 0,8\end{array}$ \\
\hline Weiser \& Godoy (2001) & 46,8 & 13,5 & 2,1 & 18,4 & 1,4 & 5,7 & $\begin{array}{l}\text { Lianas: } 10,6 \\
\text { Epífitos: } 1,4\end{array}$ \\
\hline
\end{tabular}


Formas de vida - O espectro biológico do cerrado da chapada do Araripe foi representado por 54 (50,7\%) fanerófitos, 16 (14,9\%) hemicriptófitos, 14 (13,1\%) caméfitos, 10 (9,3\%) terófitos, quatro (3,7\%) fanerofitocaméfitos, quatro (3,7\%) geófitos, três (2,8\%) epífitos e dois $(1,8 \%)$ hemiparasitos (Tab. 1$)$.

\section{Discussão}

Levantamento florístico - Dentre as famílias de maior riqueza encontradas neste trabalho, Fabaceae, Myrtaceae e Malpighiaceae, já haviam sido referidas por Eiten (1972) como as de maior riqueza de espécies dos cerrados brasileiros. O. Cavassan (dados não publicados), Felfili \& Silva Jr. (1993), Batalha \& Mantovani (2000), Costa \& Araújo (2001) e Weiser \& Godoy (2001) também se referiram a estas famílias como as de maior riqueza no estrato arbustivo-arbóreo, resultado também encontrado neste trabalho. Entretanto, Vochysiaceae, que está entre as de maior riqueza de espécies nos cerrados do Centro-Oeste e Sudeste (Felfili \& Silva Jr. 1993; Costa \& Araújo 2001; Weiser \& Godoy 2001), no cerrado da chapada do Araripe foi representada por uma única espécie, Qualea parviflora. No componente herbáceosubarbustivo, as famílias Asteraceae, Cyperaceae e Poaceae são também de maior riqueza de espécies dos cerrados do Centro-Oeste (Filgueiras \& Pereira 1990) e Sudeste (Batalha \& Mantovani 2000).

Quanto à riqueza de espécies do componente arbustivo-arbóreo (59 espécies) do cerrado da chapada do Araripe, verificou-se que, em geral, foi maior que a riqueza de espécies das disjunções de cerrado em regiões com maior precipitação como na Amazônia, oeste do Pantanal e Sul do Brasil, exceto nos cerrados do Amapá (Sanaiotii et al. 1997), que apresentou número de espécies equivalente a deste trabalho (61 espécies). Vale ressaltar que no cerrado do Amapá os autores apresentaram listagem geral que incluiu levantamentos em 11 locais diferentes. Portanto, é possível que a diversidade alfa seja menor, reforçando neste caso, a opinião de Ratter et al. (1997). Comparando este estudo com os cerrados da área nuclear e marginal, no planalto Central, Minas Gerais, Goiás e Distrito Federal (Felfili \& Silva Jr. 1993) e no cerrado do Piauí (Castro et al. 1998), onde foram encontradas de 55 a 76 espécies, e os cerrados de São Paulo (O. Castro, dados não publicados; Pagano et al. 1989) (Tab. 3), com 83 e 123 espécies, respectivamente, o cerrado do Araripe apresentou menor número de espécies. Isto era esperado, pelo fato de estar inserido em um clima semi-árido e distante da área core. Porém, a diversidade de critérios de inclusão utilizados nos trabalhos (Tab. 3) dificulta uma análise mais consistente, como já ressaltado por A.A.J.F. Castro (dados não pulicados).

Dentre as 12 espécies de ampla distribuição geográfica, Bowdichia virgilioides e Byrsonima verbascifolia já haviam sido citadas por Eiten (1972) como comuns no cerrado e de ampla distribuição, ocorrendo até nas savanas amazônicas e norte da América do sul. As análises realizadas por Ratter et al. (2003) em 376 áreas de cerrado e savanas amazônicas, revelaram que oito espécies (67\%) das 12 aqui citadas têm ocorrência em pelo menos 50\% dos sítios por eles analisados. São elas: B. virgilioides (em 77\%), Byrsonima coccolobifolia (65\%), B. verbascifolia (50\%), Hancornia speciosa (53\%), Hymenaea stignocarpa (73\%), Qualea parviflora (78\%), Tocoyena formosa (58\%) e Vatairea macrocarpa (59\%).

Padrões fenológicos reprodutivos e síndromes de dispersão - A maior proporção de espécies florescendo e frutificando durante a estação chuvosa (76\%) encontrada neste estudo, também foi observada por Mantovani \& Martins (1988), Batalha et al. (1997), Batalha \& Mantovani (2000) e Weiser \& Godoy (2001), em diferentes áreas de cerrado do Estado de São Paulo.

Dentre as espécies que não floresceram e não frutificaram $V$. macrocarpa e Platymenia reticulata (Fabaceae) também não foram encontradas em floração ou frutificação por Mantovani \& Martins (1988) em São Paulo e Miranda (1995) no Pará, respectivamente. A ocorrência de espécies com padrão de floração supra-anual e subanual (Newstrom \& Franquie 1994) no cerrado da chapada do Araripe e supra-anual nos cerrados estudados por Mantovani \& Martins (1988) e Miranda (1995) confirmam as observações de Laboriau (1963), de que algumas espécies de cerrado não florescem todos os anos, e outras mais de uma vez por ano. A produção simultânea de flores e frutos encontrada neste estudo também foi verificada por Weiser \& Godoy (2001) e Mantovani \& Martins (1988).

Formas de vida - As proporções de fanerófitos, caméfitos e hemicriptófitos encontradas foram similares às observadas por Weiser \& Godoy (2001), no Parque Estadual do Vaçununga, no Estado de São Paulo (Tab. 5). Porém, nos cerrados estudados por Batalha et al. (1997) e Mantovani \& Martins (1993), hemicriptófito foi a forma de vida dominante, possivelmente deve-se ao fato daquelas áreas 
apresentarem fisionomias mais abertas, com um estrato herbáceo abundante. Quanto às percentagens das formas de vida geófitos e terófitos, estas foram similares entre os trabalhos (Tab. 5), independente da maior aridez no Araripe e da fisionomia mais aberta como nos cerrados estudados por Batalha et al. (1997) e Mantovani \& Martins (1993).

Devido à diversidade de critérios utilizados nos levantamentos de cerrado publicados até o momento e à variação de fisionomia, não foi possível afirmar se a menor riqueza de espécies do cerrado da chapada do Araripe, em relação à área core, foi em função do isolamento geográfico e das condições climáticas mais áridas.

A escassez de levantamentos florísticos nos cerrados do Nordeste, apenas quatro incluindo este estudo, dificulta análise mais consistente do padrão de distribuição das espécies do cerrado da chapada do Araripe. Portanto, a ocorrência exclusiva de 27,6\% das espécies neste estudo não pode ser indicativo de endemismos na área.

Quanto aos aspectos fenológicos reprodutivos e síndromes de dispersão das espécies, em geral, foram similares aos já encontrados nos cerrados de São Paulo. Com base nos trabalhos analisados, a proporção de formas de vida temporárias parece estar mais relacionada à fisionomia que às variações de precipitação.

\section{Agradecimentos}

Ao CNPq, pela concessão da bolsa de Iniciação Científica do primeiro autor; ao IBAMA/Crato, pela liberação para a realização deste trabalho na Área da Floresta Nacional do Araripe; às professoras Carolyn Proença e Lúcia Helena Soares e Silva da UnB, pelo apoio na identificação das espécies de Myrtaceae; aos Biólogos Expedito Rômulo Amado de Oliveira, pela identificação das Cyperaceae; à Sarah Sued, pelo apoio na coleta e herborização do material coletado; ao auxiliar de campo, Sr. Antonio Boreto, pelo acompanhamento e aprendizado durante o período de coletas.

\section{Referências bibliográficas}

Araújo, G.M.; Nunes, J.J.; Rosa, A.G. \& Resende, E.J. 1997. Estrutura comunitária de vinte áreas de cerrado residuais no município de Uberlândia. Daphne 7(2): 7-14.

Bastos, M.N.C. 1984. Levantamento florístico dos campos do Estado do Pará. I- Campo de Joanes (Ilha de Marajó). Boletim do Museu Paraense Emílio Goeldi, Botânica 1 (1/2): 67-86.
Batalha, M.A. \& Mantovani, W. 2000. Reproductive phenological patterns of cerrado plant species at the Pé-de Gigante Reserve (Santa Rita do Passa Quatro, SP, Brazil): a comparation between the herbaceus and woody floras. Revista Brasileira de Biologia 60(1): 129-145.

Batalha, M.A.; Aragaki, S. \& Mantovani, W. 1997. Variações fenológicas das espécies do cerrado em Emas (Pirassununga, SP). Acta Botanica Brasilica 11(1): 61-78.

Castro, A.A.J.F. 1999. Cerrados no nordeste do Brasil: caracterização, biodiversidade e desastres. Publicações avulsas em Ciências ambientais 1: 1-19.

Castro, A.A.J.F.; Martins, F.R. \& Fernandes, A.G. 1998. The woody flora of cerrado vegetation in the state of Piauí, Northeastern Brazil. Edinburg Journal of Botany 55(3): 455-472.

Castro, A.A.J.F.; Martins, F.R.; Tamashiro, J.Y. \& Shepherd, G.H. 1999. How rich is the flora of brazilian cerrados? Annals of Missouri Botanical Garden 86: 192-224

César, O.; Pagano, S.N.; Leitão-Filho, H.F.; Monteiro, R.; Silva, O. A.; Marinis, G. \& Shepherd, G.J. 1988. Estrutura fitossociológica do estrato arbóreo de uma área de vegetação de cerrado no município de Corumbataí (Estado de São Paulo). Naturalia 13: 91-101.

Costa, A.A. \& Araújo, G.M. 2001. Comparação da vegetação arbórea de cerradão e de cerrado na Reserva do Panga, Uberlândia, Minas Gerais. Acta Botanica Brasilica 15(1): 63-72.

Coutinho, L.M. 1978. O conceito de cerrado. Revista Brasileira de Botânica 1: 17-23

Cronquist, A. 1981. An integrated system of classification of flowering plants. New York, Columbia University Press.

Dantas, M. \& Rodrigues, I.A. 1982. Estudos fitoecológicos do trópico úmido brasileiro: IV - Levantamentos botânicos em Campos do Rio Branco. Boletim de Pesquisa, EMBRAPA 40: 1-31.

Egler, W. 1960. Contribuições ao conhecimento dos campos da Amazônia. I - Os campos do Ariramba. Boletim do Museu Paraense Emilio Goeldi 4: 1-40.

Eiten, G. 1972. The cerrado vegetation of Brazil. Botanical Review 38(2): 201-341

Felfili, J.M. \& Silva Jr., M.C. 1993. A comparative study of cerrado (sensu stricto) vegetation in Central Brazil. Journal of Tropical Ecology 9: 277-289.

Felfili, J.M.; Silva Jr., M.C.; Rezende, A.V.; Machado, J.W.B.; Walter, B.M.T.; da Silva, P.E.N. \& Hay, J.D. 1993. Análise comparativa da florística e fitossociologia da vegetação arbórea do cerrado sensu strictu na chapada Pratinha, DF, Brasil. Acta Botanica Brasilica 6(2): 27-46.

Fernandes, A. 1990. Temas fitogeográficos. 2 ed. Fortaleza, Stylus Comunicações.

Figueiredo, M.A. 1989. Nordeste do Brasil - Relíquias vegetacionais no semi-árido cearense (Cerrados). Coleção Mossoroense, Série B, 646, Mossoró, ESAM.

Figueiredo, M.A. 1997. Unidades Fitoecológicas. In: Atlas do Ceará, Fortaleza, Ed. IPLANCE.

Filgueiras, T. \& Pereira, B.A.S. 1990. Flora do Distrito Federal. Pp. 331-388. In: M.N. Pinto (org.). Cerrado: caracterização, ocupação e perspectivas. Brasília, EUnB/ SEMATEC. 
Furley, P.A. \& Ratter, J.A. 1988. Soil resources and plant communities of central Brazilian cerrado and their development. Journal of Biogeography 15: 97-108.

Gottsberger, G. \& Moravetz, W. 1986. Floristic, structural and phytogeographical analysis of the Savannas of Humaitá (Amazonas). Flora 178: 41-71.

Jacomine, P.K.T.; Almeida, J.C. \& Medeiros, L.A.R. 1973. Levantamento exploratório - Reconhecimento de solos do Estado do Ceará. v.I. Recife.

Labouriau, L.G. 1963. Problemas de fisiologia ecológica dos cerrados. In: M.G. Ferri (coord.). I Simpósio sobre o cerrado. São Paulo, Edgard Blucher e EDUSP.

Mantovani, W. \& Martins, F.R. 1988. Variações fenológicas das espécies do cerrado da Reserva Biológica de Moji Guaçu, Estado de São Paulo. Revista Brasileira de Botânica 11: 101-112.

Mantovani, W. \& Martins, F.R. 1993. Florística do cerrado na Reserva Biológica de Moji Guaçu, SP. Acta Botanica Brasilica 7(1): 33-60.

Maury, M.C.; Ramos, A.E. \& Oliveira, P.E. 1994. Levantamento florístico da Estação Ecológica de Águas Emendadas. Boletim do Herbário Ezechias Paulo Heringer 1: 46-67.

Miranda, I.S. 1995. Fenologia do estrato arbóreo de uma comunidade de cerrado em Alter-do-Chão, PA. Revista Brasileira de Botânica 18(2): 235-240.

Miranda, I.S. 1993. Estrutura do estrato arbóreo do cerrado amazônico em Alter-do-Chão. Revista Brasileira de Botânica 16(2): 143-150.

MMA, Ministério do Meio Ambiente. 1999. Ações prioritárias para a conservação da biodiversidade do Cerrado e Pantanal. Brasília, MMA/FUNATURA/ Conservation International/Fund. Biodiversitas/ UnB.

Nascimento, M.T. \& Saddi, N. 1992. Structure and floristic composition in an area of cerrado in Cuiabá - MT, Brazil. Revista Brasileira de Botânica 15(1): 47-55.

Newstrom, L.E. \& Frankie, G.W. 1994. A new classification classification for plant phenology based on flowering patterns in lowland tropical rain forest trees at La Selva, Costa Rica. Biotropica 26(2): 141-159.

Oliveira-Filho, A.T. \& Carvalho, D.A. 1993. Florística e fisionomia da vegetação no extremo norte do litoral da Paraíba. Revista Brasileira de Botânica 16(1): 115-130.
Pagano, S.; Cesar, O. \& Leitão-Filho, H.F. 1989. Estrutura fitossociológica do estrato arbustivo-arbóreo da vegetação de cerrado da Área de Proteção Ambiental (APA) de Corumbataí - Estado de São Paulo. Revista Brasileira de Biologia 49(1): 49-59.

Pijl, Van der. 1982. Principles of dispersal in higher plants. 3 ed. New York, Springer-Verlag.

Rankiauer, C. 1934. The Life Forms of Plants and Statistical Plant Geography. Oxford, Clarendon Press.

Ratter, J.A.; Bridgewater, S. \& Ribeiro, J.F. 2003. Analysis of the floristic composition of the Brazilian cerrado vegetation III: comparasion of the woody vegetations of 376 areas. Edinburgh Journal of Botany 60(1): 57-109.

Ratter, J.A.; Ribeiro, J.F. \& Bridgewater, S. 1997. The brazilian cerrado vegetation and threats to its biodiversity. Annals of Botany 80: 223-230.

Rodrigues W.A. 1971. Plantas dos Campos do Rio Branco (Território de Roraima). In: M.G. Ferri (coord.). III Simpósio sobre o cerrado. São Paulo, Edgard Blucher e EDUSP.

Sanaiotti, T.; Bridgewater, S. \& Ratter, S.A. 1997. A floristic study of the savanna vegetation of the state of Amapá, Brazil, and suggestions for its conservation. Boletim do Museu Paraense Emílio Goeldi, Série Botânica 13(1): 3-29.

Sarmento, A.C. \& Soares, C.M.C. 1971. Nova área de cerrado em Pernambuco. Anais do ICB - Universidade Federal Rural de Pernambuco, Recife, 1(1): 75-82.

SUDENE. 1982. Climatologia e Balanço Hídrico. Ceará. Dados pluviométricos, temperaturas estimadas por regressão. Projeto: Delimitação e Regionalização do Brasil semi-árido.

Takeuchi, M. 1960. A estrutura da vegetação da Amazônia. II - As Savanas do norte da Amazônia. Boletim do Museu Paraense Emílio Goeldi 7: 1-18.

Tavares, S. 1988. Contribuição para o estudo da cobertura vegetal dos tabuleiros do nordeste. Coleção Mossoroense, Série B, n. 494.

Uhlmann, A.; Galvão, F. \& Silva, S.M. 1998. Análise da estrutura de duas unidades fitofisionômicas de Savanna (Cerrado) no sul do Brasil. Acta Botanica Brasilica 12(3): 231-247.

Weiser, V.L. \& Godoy, S.A.P. 2001. Florística em um hectare de cerrado sensu stricto na ARIE - Cerrado Pé-deGigante, Santa Rita do Passa Quatro, SP. Acta Botanica Brasilica 15(2): 201-212. 